\title{
A theoretical study of the propagation of light soliton produced by semiconductor quantum dots through optical fibers
}

\author{
O.P. Swami ${ }^{1}$, V. Kumar ${ }^{2}$, B. Suthar ${ }^{3}$, A. K. Nagar ${ }^{2}$ \\ ${ }^{1}$ Department of Physics, Government College, Loonkaransar, Bikaner, Rajasthan, 334001, India \\ ${ }^{2}$ Department of Physics, Government Dungar College, Bikaner, Rajasthan, 334001, India \\ ${ }^{3}$ Department of Physics, MLB Government College, Nokha, Bikaner, Rajasthan, 334803, India
}

omg1789@gmail.com, vijendrasaini2009@gmail.com, bhuvneshwer@gmail.com, ajaya.nagar@gmail.com

PACS 42.65.-k, 42.65.Tg, 78.67.Hc

DOI 10.17586/2220-8054-2019-10-3-273-281

\begin{abstract}
In this paper, the propagation of light soliton is studied in nonlinear optical fiber. We propose the external excitation of semiconductor quantum dot (SQD) waveguides through an optical source which allows the generation of solitonic pulses. These solitonic pluses are propagated through a nonlinear optical fiber. Soliton formation is investigated by the interaction between the external optical excitation and SQDs. Here, the SQDs are considered as a quantum system of three energy levels. In this study, the Fourier Split-Step (FSS) method is used to solve the numerically continuous nonlinear Schrdinger equation (NLSE) for evolution of the soliton pulse emitted by the SQDs inside an optical fiber with real physical parameters. The effect of a SQD's density and electric field on the pulse width is also studied. Phase plane portraits are drawn to observe the stability of soliton in fiber and SQDs.
\end{abstract}

Keywords: quantum dots, nonlinear optics, optical solitons, nonlinear guided waves.

Received: 13 March 2019

Revised: 1 April 2019

Final revision: 10 May 2019

\section{Introduction}

Semiconductor quantum dots (SQDs) are model systems for investigating nonlinear light-matter interaction that have attracted much interest. SQDs are referred to zero-dimensional systems that restrict the movement of charge carriers in the three spatial dimensions, which results in atom-like discrete energy spectra and strongly enhanced carrier lifetimes [1]. These atoms like structures have been proposed for the use as qubits in quantum information processing [2] as well as for laser devices [3]. Some of the most recent investigations indicate that these types of heterostructures can undergo abrupt changes in the spectral response with minimal variations in their size and morphology, offering important applications for optics, among which are next-generation lasers, diodes, light emitters, optical multiplexers, biosensors, spectral tuners, quantum computing, logic gates, among others [4-12]. Due to their large dipole moments, reaching values on the order of $10^{-17}$ esu $\mathrm{cm}$ [13], the interaction between SQDs and optical light fields is strongly enhanced in comparison with atomic systems, making them good candidates for the study of nonlinear optical propagation effects. Recently, it has become possible to combine nanostructures with other polymeric materials such as optical fibers, giving rise to nanocomposites, which are generally composed of several phases such as $\mathrm{SiO}_{2}$, where one or more of its dimensions are found at the nanoscale [14-22]. The optical fibers are important elements for the propagation of light wave signals with water and for the propagation of electromagnetic waves in the visible, ultraviolet and infrared range. In an optical fiber, it is necessary to consider a theory of wave propagation in dispersive media. The nonlinear Schrödinger equation (NLSE) gives a complete description of a variety of the localized non-linear effects that have been extensively studied in various contexts of the sciences and that the theory can directly link with the propagation of intense optical pulses in non-linear optical fibers that give rise to the optical solitons. Although there are many experimental advances in the propagation of solitons in optical fibers, in practice, the propagation of solitary pulses across large distances at a commercial level has not been possible due to the different technical functions [23-26]. The study allows us to propose a general model which permits the coupling of solitons from a set of quantum dots with specific characteristics in an optical fiber that has non-linear optical characteristics. As a result, a numerical simulation is developed to study the evolution of the soliton inside a non-linear optical fiber with the Fourier Split-Step numerical technique with the real parameters associated with the SQD and the optical fiber. The effect of soliton density and electric field on the full width at half maximum (FWHM) of re-emitted solitonic pulse is studied. Phase planes represent the stability/instability of solitons. 


\section{Theoretical analysis}

SQDs can confine the movement of charge carriers in all dimensions due to their zero dimensional nanosized structures, which exhibits a discrete energy system. We consider a two-dimensional sheet of inhomogeneously broadened SQDs which forms a transition layer on one of the surfaces of the planar waveguide. The waveguide soliton satisfies the Maxwell-Bloch equations with nonlinear boundary conditions. The purpose of the present article is to theoretically investigate the formation processes of optical solitons under the condition of SIT in a semiconductor waveguide.

A SQD of three-level energy system is considered, whose ground state is $\left|\psi_{1}\right\rangle$ and has energy $\varepsilon_{\psi_{1}}=0$. The states $\left|\psi_{2}\right\rangle$ and $\left|\psi_{3}\right\rangle$ have energies $\varepsilon_{\psi_{2}}=h v_{0}=\varepsilon_{x}+\frac{\delta_{x}}{2}$ and $\varepsilon_{\psi_{3}}=h v_{0}^{\prime}=2 \varepsilon_{x}-\delta_{x x}$, respectively. Where the quantities $\varepsilon_{x}=\left(\varepsilon_{\psi_{2}}+\varepsilon_{\psi_{2}}^{\prime}\right) / 2$ and $\varepsilon_{\psi_{3}}$ describe the energies of the single-excitonic and biexcitonic states, respectively.

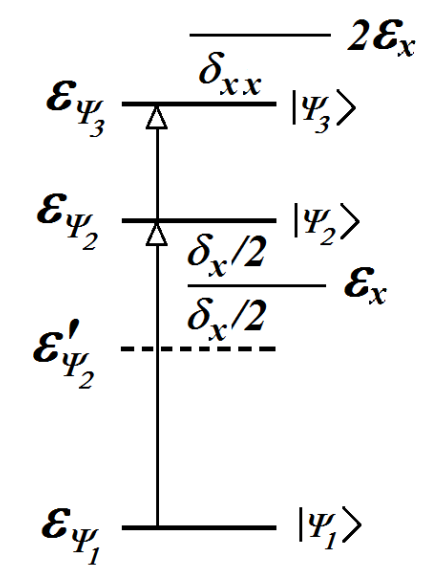

FIG. 1. Schematic diagram of energy structures of semiconductor quantum dots

The energy of exciton fine structure splitting is denoted by $\delta_{x}=\varepsilon_{\psi_{2}}-\varepsilon_{\psi_{2}}^{\prime}$ and biexciton binding energy is denoted by $\delta_{x x} . h$ is Planck constant. The Hamiltonian of system is described by:

$$
H=H_{0}+H_{0}^{\prime},
$$

where $H_{0}=h v_{0}\left|\psi_{2}\right\rangle\left\langle\psi_{2}\left|+\frac{1}{2} h v_{0}^{\prime}\right| \psi_{3}\right\rangle\left\langle\psi_{3}\right|$ is called the Hamiltonian of single excitonic state $\left|\psi_{2}\right\rangle$ and biexcitonic state $\left|\psi_{3}\right\rangle$. The additional term $H_{0}^{\prime}$ in the Hamiltonian is present due to the interaction of the light pulse with SQDs and $H_{0}^{\prime}=-\vec{P} \cdot \vec{E}$. During the excitation of the SQDs, an external light source is considered, composed of a linearlypolarized, high intensity optical wave from a laser and we will study the formation of non-linear optical waves that are re-emitted by the SQDs. Fig. 2 represents a schematic of a linearly-polarized plane wave incident on SQDs. In the process, due to the interaction of the light with the SQDs, the light is re-emitted in the form of a non-linear wave with special characteristics that will depend on the morphology of the quantum dot and the incident wave.

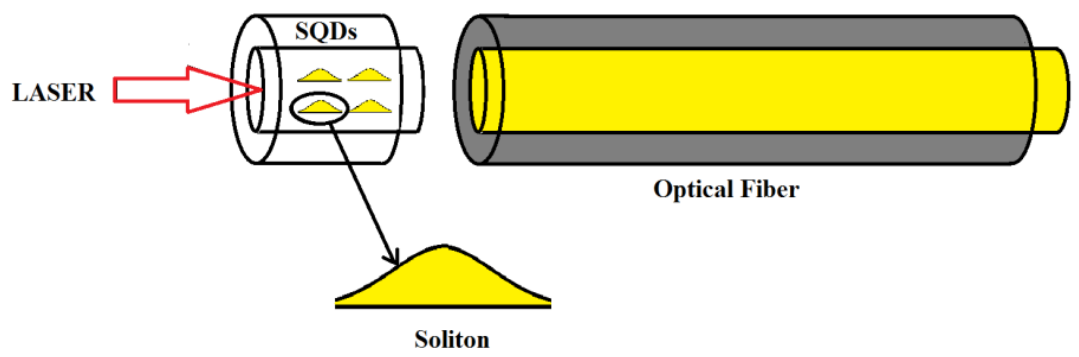

FIG. 2. Schematic arrangement of SQDs and optical fiber with an external light source 
In the analytical treatment the electric field vector of incident light beam is taken as below:

$$
\vec{E}(z, t)=\sum_{l= \pm 1} \vec{e}_{l} \hat{E}_{l} \varphi_{l},
$$

where:

$$
\vec{e}=\frac{1}{\sqrt{2}}(\vec{x}+i \vec{y})
$$

is the complex polarization vector. $\vec{x}$ and $\vec{y}$ are unit vector along the $x$ and $y$ axes. $\varphi_{l}=e^{i l\left(k z-v_{l} t\right)}$ is a periodic wave function and $\hat{E}$ is the slowly changing amplitude of the electric field. The incident light is considered linearlypolarized in TE mode and has a width $T$ and angular frequency $w \gg T-1$ propagating along $z$ direction. When an external light beam interacts with SQDs, then a secondary dipolar field is produced in same direction as incident field and can be written as:

$$
-c^{2} \frac{\partial^{2} \vec{E}(z, t)}{\partial z^{2}}+\eta^{2} \frac{\partial^{2} \vec{E}(z, t)}{\partial t^{2}}=-4 \pi \frac{\partial^{2} \vec{P}(z, t)}{\partial t^{2}},
$$

where the polarization component of ensemble of SQDs is the following:

$$
\vec{P}(z, t)=\frac{1}{2} n_{0} \int g\left(v_{01}\right) \vec{e}\left(\mu_{12} \rho_{21}+\mu_{23} \rho_{32}\right)(v, z, t) d v+c . c .,
$$

which is determined by the interband transitions occurring in the quantum dots between the three energy levels. The quantities $\mu_{12}$ and $\mu_{23}$ are the dipole moments for the corresponding transitions which we assume to be parallel to each other. $g\left(v_{01}\right)$ is an inhomogeneous broadening function which arises due to the quantum dot size fluctuations. Because the energy levels depend on the size of dots, the exciton frequency $v_{0}$ and detuning quantity $v_{01}=v_{0}-v_{1}$ are also size dependent. $\eta$ is the refractive index of semiconductor and $n_{0}$ is the density of Quantum Dots. $\rho_{i j}$ are the matrix elements of the density matrix and $\rho$ can be determined from Liouville equation:

$$
i \hbar \dot{\rho}_{m n}=\sum\left(\langle n|H| l\rangle \rho_{l m}-\rho_{n l}\langle l|H| m\rangle\right),
$$

where $n, m, l=1,2,3$.

The density matrix of the pulse is considered of the form:

$$
\rho_{21}=\hat{\rho}_{21} \varphi_{3}, \quad \rho_{32}=\hat{\rho}_{32} \varphi_{1}, \quad \rho_{31}=\hat{\rho}_{31} \varphi_{2},
$$

where $\hat{\rho}_{n m}$ is the slowly varying complex amplitudes of the elements of density matrix. $\varphi_{1,2,3}$ is taken in for envelope function. To guarantee that $E$ is a real number we choose slow envelope approach, it is considered that amplitude $\hat{E}_{l}$ of pulse is very smooth in space and time and can be written as:

$$
\left|\frac{\partial \hat{E}_{l}}{\partial t}\right| \ll v_{l}\left|\hat{E}_{l}\right|
$$

and

$$
\left|\frac{\partial \hat{E}_{l}}{\partial z}\right| \ll k\left|\hat{E}_{l}\right|
$$

then nonlinear carrier wave equation can be written as following form:

$$
\sum_{l= \pm 1} \varphi_{l}\left[l^{2}\left(c^{2} k^{2}-\eta^{2} v_{1}^{2}\right) \hat{E}_{l}-2 i k l c^{2} \frac{\partial \hat{E}_{l}}{\partial z}-2 i l \eta^{2} v_{1} \frac{\partial \hat{E}_{l}}{\partial t}\right]=-2 \pi l^{2} v_{1}^{2} n_{0}\left[\mu_{12} \hat{\rho}_{21}+\mu_{23} \hat{\rho}_{32}\right] e^{i\left(k x-v_{1} t\right)}+c . c .
$$

The diagonal elements $\rho_{12}, \rho_{22}$ and $\rho_{33}$ give rise to the populations in the states $\left|\psi_{1}\right\rangle,\left|\psi_{2}\right\rangle$ and $\left|\psi_{3}\right\rangle$ respectively. Non-diagonal elements $\rho_{m n}(n \neq m)$ contain the relative phase between the states that describe the atomic coherence. In the absence of phase modulation $\hat{E}_{l}=\hat{E}_{-l}=\hat{E}_{l}^{*}=\hat{E}$. Therefore, the system of equations for the slowly varying amplitudes:

$$
\begin{gathered}
i \hbar \dot{\rho}_{11}=\left(\hat{\rho}_{21}^{*}-\hat{\rho}_{21}\right) \mu_{12} \hat{E}, \\
i \hbar \dot{\rho}_{22}=\left(\hat{\rho}_{21}-\hat{\rho}_{21}^{*}+\delta \hat{\rho}_{32}^{*}-\delta \hat{\rho}_{32}\right) \mu_{12} \hat{E} \\
i \hbar \dot{\rho}_{33}=\left(\hat{\rho}_{32}-\hat{\rho}_{32}^{*}\right) \mu_{12} \hat{E} \\
i \hbar \dot{\rho}_{21}=h\left(v_{0}-v\right) \hat{\rho}_{21}-\left[\left(\hat{\rho}_{11}-\hat{\rho}_{22}\right)+\delta \hat{\rho}_{31}\right] \mu_{12} \hat{E}, \\
i \hbar \dot{\rho}_{32}=h\left(v_{0}^{l}-v_{0}-v\right) \hat{\rho}_{32}+\left[\delta\left(\hat{\rho}_{33}-\hat{\rho}_{22}\right)+\hat{\rho}_{31}\right] \mu_{12} \hat{E} \\
i \hbar \dot{\rho}_{31}=h\left(v_{0}^{l}-2 v\right) \hat{\rho}_{31}+\left[\hat{\rho}_{32}-\delta \hat{\rho}_{21}\right] \mu_{12} \hat{E} .
\end{gathered}
$$


In the above equations, the rotating wave approximation has been applied and $\delta=\frac{\mu_{23}}{\mu_{12}}$. The solution of the system of equations (11), allows the calculation of the elements of the right side of equation (10). As the diagonal elements of matrix give rise to the populations in the states, for ground state $\rho_{11}=1, \rho_{11}=0$ and $\rho_{33}=0$, the equations (11) take the form:

$$
\begin{gathered}
\hat{\rho}_{21}=\frac{i}{2 d^{3}}\left(\sin 2 A d+2 \delta^{2} \sin A d\right), \\
\hat{\rho}_{32}=\frac{i \delta}{2 d^{3}}(\sin 2 A d-2 \sin A d),
\end{gathered}
$$

where $d=\sqrt{\left(1+\delta^{2}\right)}$ and $A=\frac{2 \pi \mu_{12}}{h} \int_{-\infty}^{t} E(z, t) d t$, which is called the area of nonlinear optical pulse.

Applying the dispersion law for linear wave guide modes, wave equation (4) will take following form:

$$
(2 \psi)_{t t}+\frac{c}{\eta}(2 \psi)_{z t}+\frac{2 \pi^{2} v \mu_{12}^{2} n_{0}}{h \eta^{2}} \sin (2 \psi)=0
$$

where

$$
\psi=A d
$$

Equation (13) can be written in the form of electric field as below:

$$
\left(\frac{d \hat{E}}{d \xi}\right)=\frac{\hat{E}^{2}}{T^{2}}-\frac{\pi^{2} \mu_{12}^{2} \hat{E}^{4}}{h^{2}}
$$

with $\xi=t-\frac{z}{V}$ and constant phase velocity $V$. The solution of equation (15) for the envelope function has the form:

$$
\hat{E}=\frac{h}{\sqrt{2} \pi \mu_{12} T} \sec h\left(\frac{\xi}{T}\right),
$$

which is well-known solitonic solution, with width of the pulse remitted by SQD:

$$
T=\sqrt{\frac{h \eta^{2}}{4 \pi^{2} v n_{0} \mu_{12}^{2}}\left(\frac{c-\eta V}{\eta V}\right)} .
$$

These soliton became stable when the speed of light (phase velocity) in SQD media $c / \eta$ remains greater than the phase velocity $V$.

\section{Result and discussion}

The theoretical result of wave equation (4) is presented as a soliton. In other words, the excitation of SQDs from an intense nonlinear wave can produce optical solitons as a result of light-SQDs interaction. During the nonlinear interaction process, the SQDs are considered as a three-level energy quantum system, in which the optical transitions are given from the ground state to the biexcitonic and excitonic states. The allowed transitions between the ground state to the biexcitonic and excitonic states have very low dipole moment compared to the transition between the ground state and the background of the exciton band. On the other hand, the characteristics of the light re-emitted by the sheets of quantum dots in the form of optical solitons depend on the intensity of the incident light, whose minimum value to form the optical solitons can be specifically determined depending on the nature of the SQDs. In this way, the soliton remitted will depend on the refractive index of the semiconductor $\eta$, the dipole moment corresponding to the transitions between the ground state and the excitonic state or between the biexcitonic state and the excitonic state $\mu_{12}$. In order to study the propagation of short optical pulses through nonlinear optical fibers, the non-linear Schrodinger equation (NLSE) is used, which takes into account the effects of the length of the fiber, the dispersion effect of group speed and non-linear optical effects as a consequence of the high intensity of light. The Fourier Split-Step method is a pseudo-spectral technique that is extremely useful due to its rapidity and high accuracy in calculations. In general, this method obtains an approximate solution of the propagation equation, assuming that dispersion effects and non-linear effects act independently along the fiber in very small steps. This technique was used to simulate the evolution of solitons that are re-emitted by the SQD.

In the results, it is observed that as the density of SQD increases, the peak intensity also increases. Because as the number of QDs per unit volume increases, absorption will also increases. Therefore the intensity of re-emitted beam will increase. Due to this the pulse width (FWHM) will also decrease. Same concept is applies for electric field verses FWHM. In the mathematical modelling of proposed work, the interactions of QDs in the Hamiltonian are omitted. For the simulation, we have proposed SQDs with pyramidal morphology of In as manufactured with a cylindrical symmetry with the parameters $\eta=2.11, \mu_{12}=1.92 \times 10^{-28} \mathrm{C} \cdot \mathrm{m}$ and $V=1.37 \times 10^{8} \mathrm{~m} / \mathrm{s}$. 
Figures 3(a-d) represent the simulation of solitons created by SQDs arrays with different densities of SQDs according to following table. It is clear from equation (17) that the pulse width decreases as $n_{0}$ increases. In many optical fiber communications systems, FWHM is required 1 ps or below, which can be achieved by increasing the density of SQDs. Simulated data are summarized in Table 1.

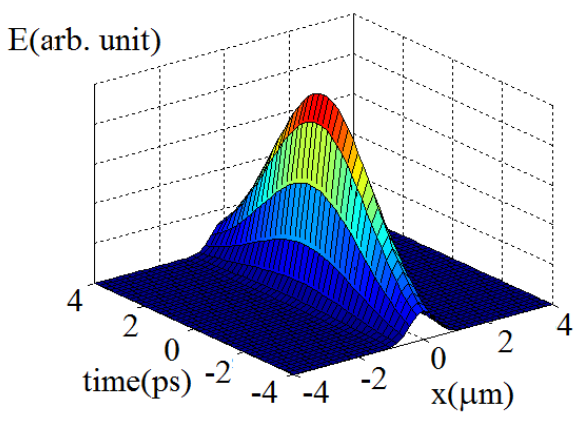

(a)

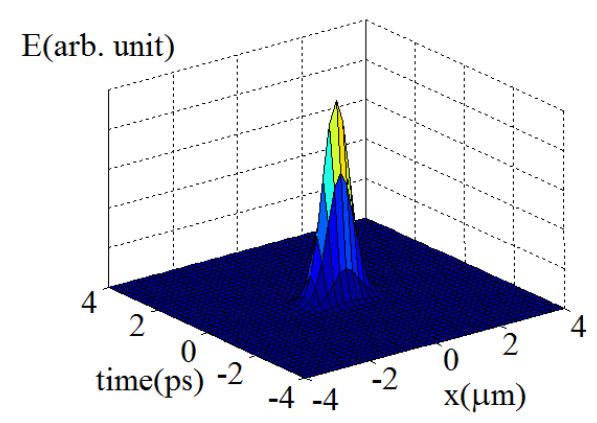

(c)

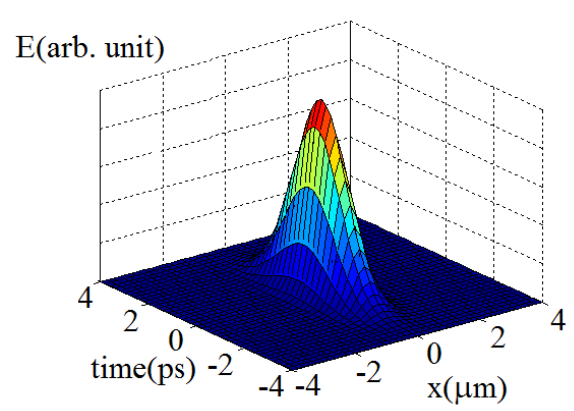

(b)

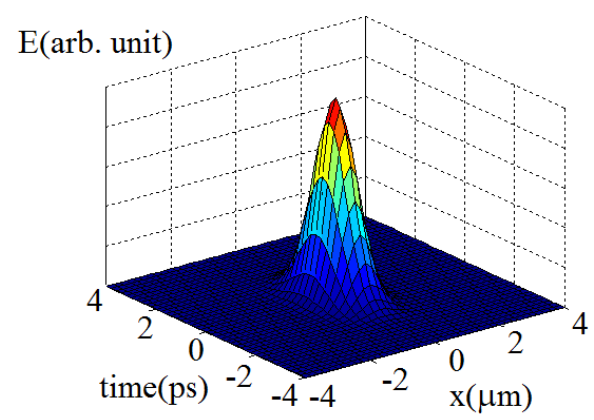

(d)

FIG. 3. Simulation of soliton generated by SQDs system with different densities of QDs.(a) $n_{0}=$ $2.59 \times 10^{12} \mathrm{~cm}^{-3}$; (b) $n_{0}=8.00 \times 10^{12} \mathrm{~cm}^{-3}$; (c) $n_{0}=23.35 \times 10^{12} \mathrm{~cm}^{-3}$ and (d) $n_{0}=$ $99.12 \times 10^{12} \mathrm{~cm}^{-3}$

TABLE 1. Relation between the density of SQDs and FWHM of solitons

\begin{tabular}{|c|c|c|}
\hline S.No. & Density of SQD $\left(10^{12} \mathrm{~cm}^{-3}\right)$ & FWHM(ps) \\
\hline \hline 1. & 2.59 & 6.18 \\
\hline 2. & 8.00 & 3.52 \\
\hline 3. & 23.35 & 2.06 \\
\hline 4. & 99.12 & 1.00 \\
\hline
\end{tabular}

Figures 4(a-d) represent that the soliton width as determined from equation (16) is decreased as electric field $E$ is increased. According to Table 2, it is clear that when the amplitude of the electric field is increased, then the FWHM of TM mode of wave decreases, which is a basic requirements for many optical fiber communications systems.

Figures 5(a-d) represent the evolution of soliton profiles in SQDs for different values of refractive index of material of SQDs as taking $V=1.37 \times 10^{8} \mathrm{~m} / \mathrm{s}$.

Figures 6(a-d) represent the phase plane portraits for Figs. 5(a-d) respectively for stability analysis. Closed limit cycles of phase plane structures clearly indicate a stable soliton, while spiral-like phase plane structures indicate an unstable soliton. It is clear from below Table 3 that as $c / \eta$ become less than $V$, the soliton become unstable, which is summarised in Table 3. 


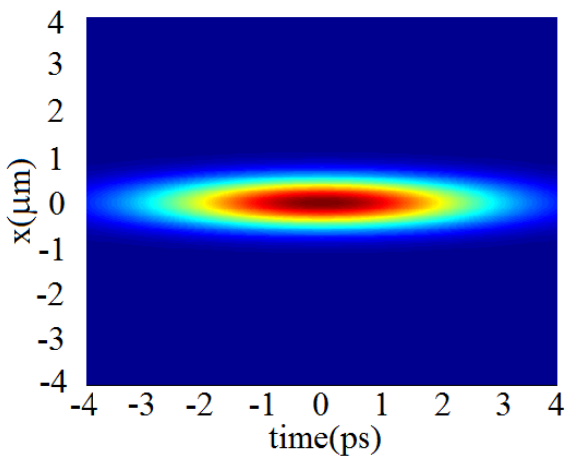

(a)

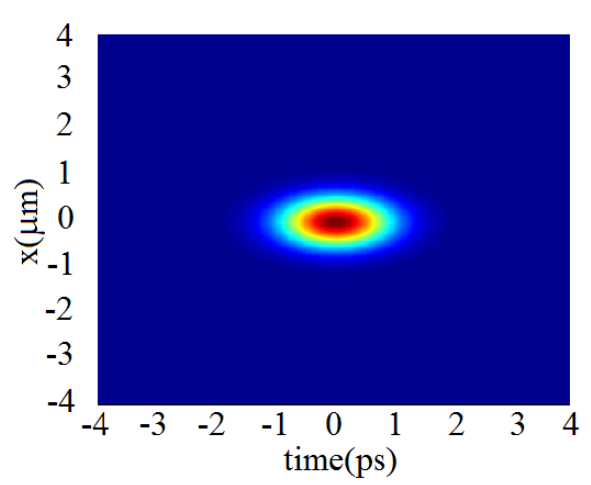

(c)

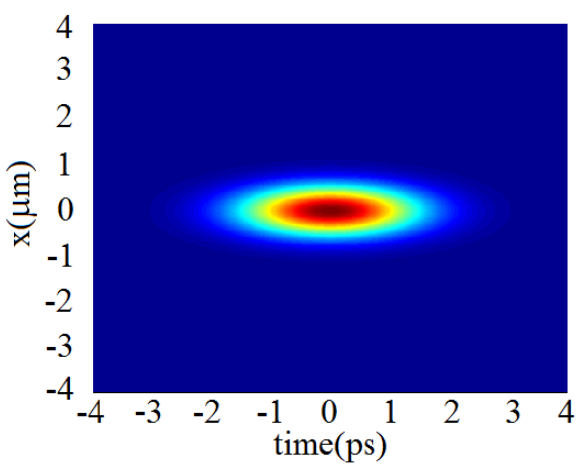

(b)

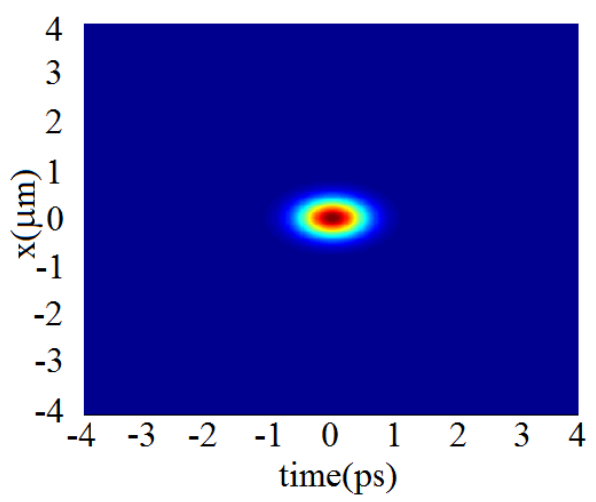

(d)

FIG. 4. Transverse view of soliton in SQD with electric field (a) $E=0.96 \times 10^{8} \mathrm{~V} / \mathrm{m}$; (b) $E=$ $1.73 \times 10^{8} \mathrm{~V} / \mathrm{m}$; (c) $E=2.86 \times 10^{8} \mathrm{~V} / \mathrm{m}$ and (d) $E=6.00 \times 10^{8} \mathrm{~V} / \mathrm{m}$

TABLE 2. Relation between the amplitude of Electric field and FWHM of TM mode of solitons

\begin{tabular}{|c|c|c|}
\hline S.No. & Amplitude of electric field $\left(10^{8} \mathrm{~V} / \mathrm{m}\right)$ & FWHM(ps) \\
\hline \hline 1. & 0.96 & 6.26 \\
\hline 2. & 1.73 & 3.46 \\
\hline 3. & 2.86 & 2.10 \\
\hline 4. & 6.00 & 1.00 \\
\hline
\end{tabular}

TABLE 3. Relation between refractive index of material of SQDs and (in)stability of soliton

\begin{tabular}{|c|c|c|c|}
\hline S. No. & Refractive index $(\eta)$ of SQDs & $c / \eta(\mathrm{m} / \mathrm{s})$ & Nature of soliton \\
\hline \hline 1 & 2.05 & $1.46 \times 10^{8}$ & Stable \\
\hline 2 & 2.11 & $1.42 \times 10^{8}$ & Stable \\
\hline 3 & 2.40 & $1.25 \times 10^{8}$ & Unsable \\
\hline 4 & 3.50 & $0.86 \times 10^{8}$ & Unstable \\
\hline
\end{tabular}




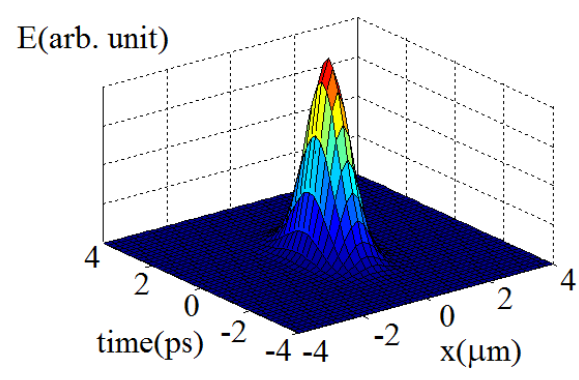

(a)

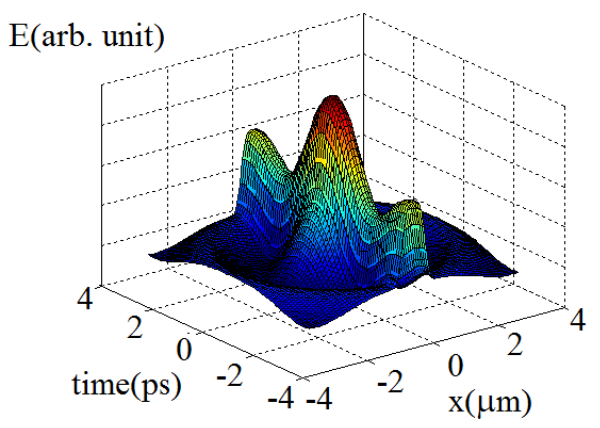

(c)

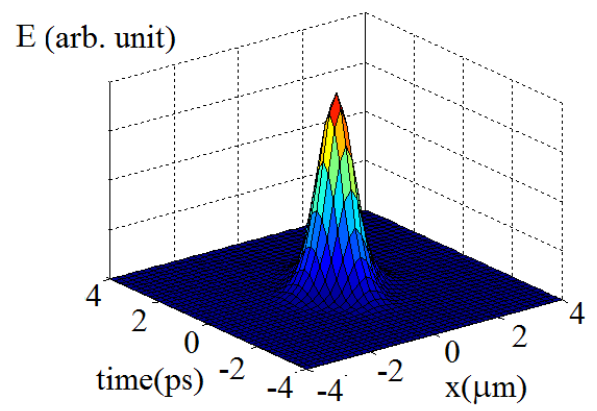

(b)

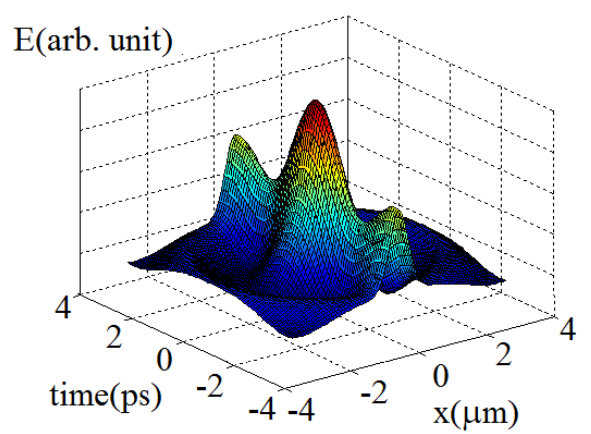

(d)

FIG. 5. Simulation of soliton generated by SQDs system with different refractive indices of QDs. (a) 2.05 ; (b) 2.11 ; (c) 2.40 and (d) 3.50 taking $V=1.37 \times 10^{8} \mathrm{~m} / \mathrm{s}$

Figure 7(a) represents the soliton profiles for input pulse in optical fiber and the Fig. 7(b) represents the pulse in optical fiber after travelling the distance of $z=1000 \mathrm{~m}$. Simulations are made using split-step Fourier method with the parameters: nonlinear fiber parameter $\gamma=0.32 \mathrm{~W} / \mathrm{m}$, fiber attenuation constant $\alpha=0.2 \mathrm{~dB} / \mathrm{km}$ and second order dispersion coefficient $\beta=-2 \times 10^{-26} \mathrm{~s}^{2} / \mathrm{m}$.

\section{Conclusions}

In this paper, we have investigated the formation of optical solitonic pulses in three level semiconductor quantum dots embedded in nonlinear optical fibers for propagation of fields without losses over long distances. Light solitons are produced in semiconductor quantum dots by the interaction of laser field with SQDs. The FWHM of solitonic pulses can be modulated according to densities of SQDs and field intensity. If the density of SQD is increased, the peak intensity is also increased. Because as the number of QDs per unit volume is increased, absorption will also be increased. Therefore the intensity of re-emitted beam will be increased. Due to this, the pulse width (FWHM) will also be decreased. The same concept is applied for electric field verses FWHM. The stability/instability of generated solitons is dependent on the refractive indices of SQDs material. Likewise, it is proposed the manufacture of these types of nanostructures with different morphologies inserted in optical fibers that allow the propagation of solitons without losses over the long distances. 


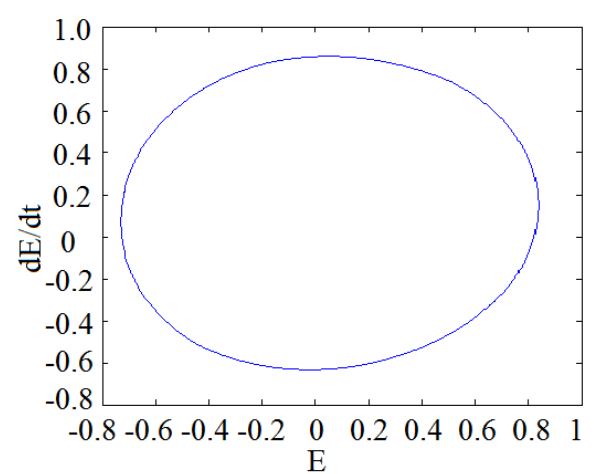

(a)

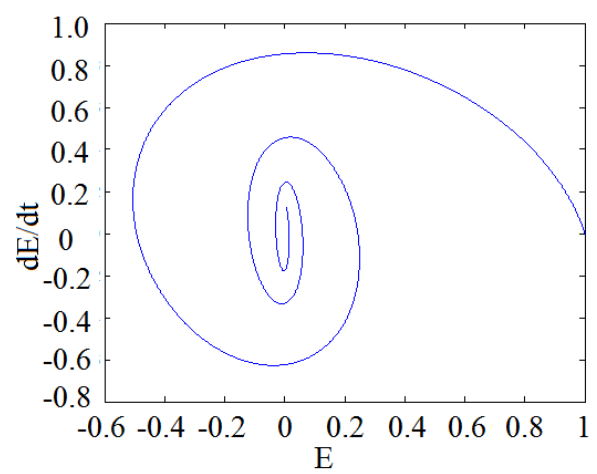

(c)

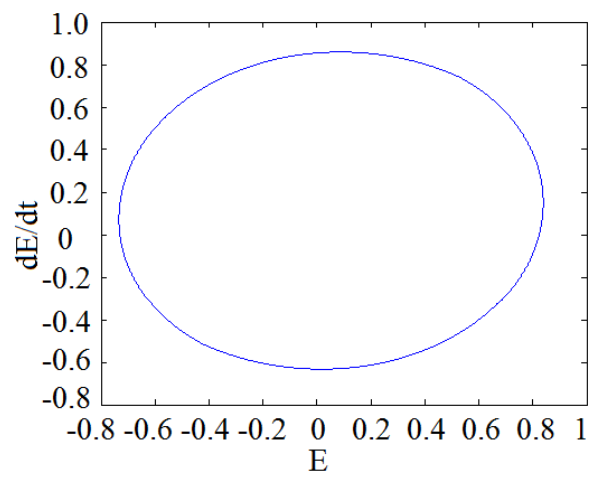

(b)

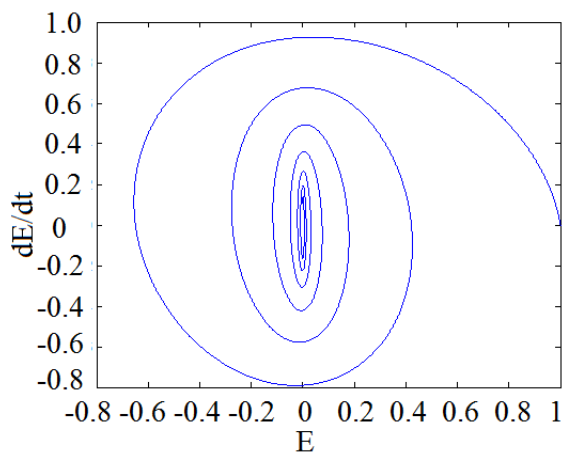

(d)

FIG. 6. Phase plane portraits for solitons for different refractive indices of QDs.(a) 2.05; (b) 2.11; (c) 2.40 and (d) 3.50 taking $V=1.37 \times 10^{8} \mathrm{~m} / \mathrm{s}$

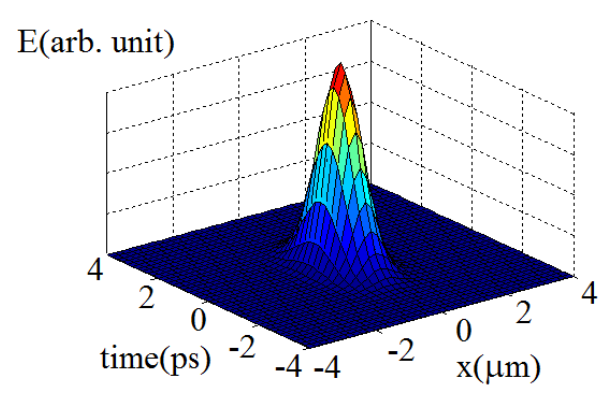

(a)

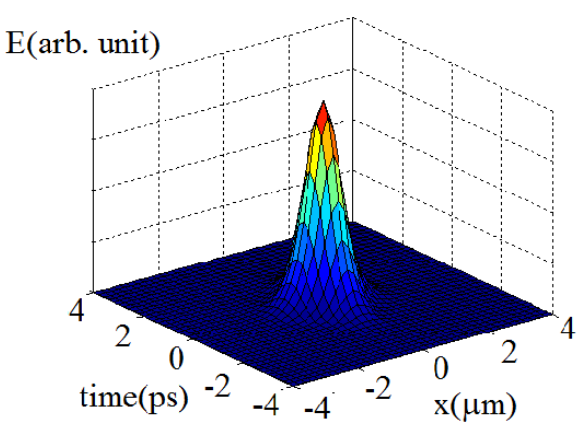

(b)

FIG. 7. Evolution of soliton through nonlinear optical fiber at (a) $z=0 \mathrm{~m}$ and (b) $z=1000 \mathrm{~m}$

\section{References}

[1] Pavesi L., Dal Negro L., et al. Optical gain in silicon nanocrystals. Nature, 2000, 408, P. 440-444.

[2] Hu Y.M., Yang W.L., Feng M., Du J.F. Distributed quantum-information processing with fullerene-caged electron spins in distant nanotubes. Phys. Rev. A, 2009, 80, P. 022322(1-13).

[3] Máximo L., Mendez V.H. Autoensamblado de puntos cunticos. Cinvestav, 2008, 27, P. 44-49.

[4] Hanewinkel B., Knorr A., Thomas P., Koch S.W. Optical near-field response of semiconductor quantum dots. Phys. Rev. B, 1997, 55, P. 13715(1-10).

[5] Aknmanov S.A., Visloukh V.A., Chirkin A.S. Optics of Femtosecond Laser Pulses. New York, American Institute of Physics, 1992.

[6] Klimov V.I., Mikhailovsky A.A., et al. Optical gain and stimulated emission in nanocrystal quantum dots. Science, 2000,290 , P. $314-317$.

[7] Yang W.X., Chen A.X., Lee R.K., Wu Y. Matched slow optical soliton pairs via biexcition coherence in quantum dots. Phys. Rev. A, 2011, 84, P. 013835(1-11). 
[8] Hu L., Wu H., et al. The effect of annealing and photoactivation on the optical transitions of band-band and surface trap states of colloidal quantum dots in PMMA. Nanotechnology, 2011, 22, P. 125202(1-8).

[9] Adamashvili G.T., Weber C., Knorr A. Optical nonlinear waves in semiconductor quantum dots: Solitons and breathers. Phys. Rev. A, 2007, 75, P. 1-9.

[10] Newell A.C. Solitons in Mathematics and Physics. Philadelphia, Society for Industrial and Applied Mathematics, 1985.

[11] Ajayan P.M., Schadler L.S., Braun P.V. Nanocomposite science and technology. Weinheim, Germany, Wiley, 2003.

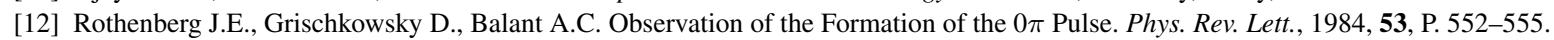

[13] Chen M., Kaup D.J., Malomed B.A. Three-wave solitons and continuous waves in media with competing quadratic and cubic nonlinearities. Phys. Rev. E, 2004, 69, P. 056605(1-17).

[14] Bimberg D., Grundmann M., Ledentsov N.N. Quantum Dot Heterostructures. Chichester, Wiley, 1999.

[15] Schneider S., Borri P., et al. Self-induced transparency in InGaAs quantum-dot waveguides. Appl. Phys. Lett., 2003, 83, P. 3668-3670.

[16] Panzarini G., Hohenester U., Molinari E. Self induced transparency in semiconductor quantum dots. Phys. Rev. B, 2002, 65, P. 165322(1-6).

[17] Klimov V.I. Nanocrystal quantum dots. Los Alamos Science, 2003, 28, P. 214-220.

[18] Chen Y., Herrnsdorf J., et al. Colloidal quantum dot random laser. Opt. Express, 2011, 19, P. $2996-3003$.

[19] Akhmediev N., Ankiewicz A. Solitons, Nonlinear pulses and Beams. London, Chapman and Hall, 1997.

[20] Kevrekidis P.G., Rasmussen K.O., Bishop A.R. Two-dimensional discrete breathers: Construction, stability, and bifurcations. Phys. Rev. E, 2000, 61, P. 2006(1-7).

[21] Blum K. Density Matrix Theory and Applications, 2nd ed. New York, Plenum Press, 1996.

[22] Adamashvili G.T., Weber C., Knorr A. Optical solitons in semiconductor Quantum dots. Eur. Phys. J. D, 2008, 47, P. $113-117$.

[23] Malomed B.A., Kevrekidis P.G. Discrete vortex solitons. Phys. Rev. E, 2001, 64, P. 026601(1-6).

[24] Lamb Jr.G.L. Elements of soliton theory. New York, Wiley, 1980.

[25] Lamb Jr.G.L. Optical waves in crystals. New York, Wiley, 1980.

[26] Oster M., Johansson M. Stable stationary and quasiperiodic discrete vortex breathers with topological charge $S=2$. Phys. Rev. E, 2006, 73, P. 066608(1-6). 